\title{
Experiências vivenciadas em atendimentos de medicina e enfermagem do SUS: reflexões sobre acesso e atenção à saúde de migrantes internacionais
}

\author{
Experiences lived in SUS medical and nursing care: reflections on access and health care \\ for international migrants
}

Experiencias vividas en en la atención médica y de enfermería del SUS: reflexiones sobre el acceso y la atención de la salud de los migrantes internacionales

Ana Clara Borges Carvalho ${ }^{*}$, Ana Júlia Araújo de Carvalho ${ }^{1}$, Lívia Lara Teodoro1, Vivianne Peixoto da Silva1.

\section{RESUMO}

Objetivo: Apresentar os entraves encontrados por migrantes internacionais, no acesso e atendimento em serviços de saúde. Relato de experiência: As experiências relatadas foram vivenciadas por estudantes de graduação, dos cursos de Medicina e Enfermagem em um Hospital de Clínicas Universitário e nas UBS de uma cidade de Minas Gerais. Os atendimentos permitiram identificar três pontos que merecem destaque, quais sejam: falhas na comunicação como uma via de mão dupla (profissional-usuário); perda de autonomia nos processos decisórios de saúde (principalmente das mulheres) e, inabilidade com competências culturais por parte dos profissionais, que podem gerar impactos nas condutas de promoção, prevenção e proteção da saúde. Considerações finais: Além do acesso, questiona-se a qualidade dos serviços de saúde prestados aos migrantes internacionais, no tocante às demandas apresentadas e sua resolutividade. Ações como políticas públicas específicas para migrantes internacionais, contratação destes nos serviços de saúde, mudanças no processo formativo dos profissionais da saúde, para inserção de competências culturais e, capacitação profissional, nos parecem boas possibilidades como mecanismos de enfrentamento conjunto para garantir que os direitos humanos sejam cumpridos sem distinção de nacionalidade, em especial, o direito universal à saúde, foco desse manuscrito.

Palavras-chave: Migração humana, Saúde, Direitos humanos.

\begin{abstract}
Objective: To present the obstacles encountered by international migrants, in access and assistance in health services. Experience report: The experiences reported were lived by undergraduate students, from Medicine and Nursing courses at a University Hospital and in the UBS of a city of Minas Gerais. The consultations allowed us to identify three points that deserve to be highlighted, namely: communication failures as a twoway street (professional-user); loss of autonomy in health decision-making processes (mainly for women) and, inability with cultural skills on the part of professionals, which can impact on the promotion, prevention and protection of health. Final considerations: In addition to access, the quality of health services provided to international migrants is questioned, with regard to the demands presented and their resolution. Actions such as specific public policies for international migrants, hiring them in health services, changes in the training process for health professionals, for the insertion of cultural skills and professional training, seem to us to be good possibilities as mechanisms for joint coping to ensure that human rights are fulfilled without distinction of nationality, in particular, the universal right to health, the focus of this manuscript
\end{abstract}

Keywords: Human migration, Health, Human rights.

1 Universidade Federal de Uberlândia (UFU), Uberlândia - MG. *E-mail: vivianne@ufu.br 


\section{RESUMEN}

Objetivo: Presentar los obstáculos que encuentran los migrantes internacionales, en el acceso y asistencia en los servicios de salud. Informe de experiencia: Las experiencias reportadas fueron vividas por estudiantes de pregrado, de cursos de Medicina y Enfermería en un Hospital Universitario y en la UBS de una ciudad. de Minas Gerais. Las consultas permitieron identificar tres puntos que merecen ser destacados, a saber: fallas de comunicación como vía de doble sentido (usuario profesional); pérdida de autonomía en los procesos de toma de decisiones en salud (principalmente de las mujeres) y, incapacidad con las habilidades culturales por parte de los profesionales, lo que puede incidir en la promoción, prevención y protección de la salud. Consideraciones finales: Además del acceso, se cuestiona la calidad de los servicios de salud brindados a los migrantes internacionales, en cuanto a las demandas presentadas y su resolución. Acciones como políticas públicas específicas para los migrantes internacionales, su contratación en los servicios de salud, cambios en el proceso de formación de los profesionales de la salud, para la inserción de competencias culturales y la formación profesional, nos parecen buenas posibilidades como mecanismos de afrontamiento conjunto para asegurar que los derechos humanos se cumplen sin distinción de nacionalidad, en particular, el derecho universal a la salud, tema central de este manuscrito

Palabras clave: Migración humana, Salud, Derechos humanos.

\section{INTRODUÇÃO}

Nos últimos anos, o Brasil tem sido um país atrativo para novos fluxos migratórios, principalmente de refugiados em razão de perseguições políticas-ideológicas, sociais e econômicas, que os expõem a condições de risco à própria vida e, a de sua família, bem como de imigrantes que deixam seu país de origem em busca de melhores condições de vida, trabalho e renda (SILVA VP, 2017; SILVA VP e SILVEIRA C, 2018).

Saladini APS (2011) destaca o aumento no fluxo de imigrantes desde a década de 1980, caracterizado por nacionalidade africana, principalmente por fuga dos efeitos da guerra civil em Angola, e, o contingente de estrangeiros de outras nacionalidades que chegam ao país a partir de 2008, com incremento especialmente de nacionais do Haiti, a partir de 2010 (PATARRA NL, 2005, 2012; RIZEK CS ET AL, 2010).

Segundo o censo demográfico do ano de 2010 realizado pelo Instituto Brasileiro de Geografia e Estatística (IBGE), a migração internacional no Brasil aumentou cerca de 90\% em comparação ao censo de 2000. Esse aumento pode ser explicado pela retomada do crescimento do Brasil advinda da estabilidade econômica e, pela crise internacional de 2008, que atingiu os países centrais (OLIVEIRA AT, 2013; FIGUEREDO LO, ZANELATTO JH, 2016).

A inserção do Brasil na agenda internacional levou a um considerável aumento do número de imigrantes oriundos de países desenvolvidos para "cargos especializados com forte exigência de mão de obra qualificada", como: portugueses, japoneses, italianos, estadunidenses, dentre outros. De forma concomitante, atraiu imigrantes de países menos desenvolvidos, como os nacionais da Bolívia, Argentina, Uruguai, Chile, Peru, Colômbia e Paraguai, com menor qualificação (GOLDBERG A, et al., 2015).

Nos anos seguintes, o Brasil seguiu a tendência já estabelecida, com crescimento contínuo em recepção de imigrantes, de acordo com o World Migration Report 2018 da Organização Internacional para as Migrações (OIM), alcançando 1\% da população total do Brasil em 2016. Dados do Comitê Nacional para os Refugiados, (Conare) apontam cerca de 11.231 pessoas reconhecidas como refugiados no Brasil em 2019 e 1,1 milhões de imigrantes segundo a EBC agência do Brasil (RIBEIRO, V, 2019).

Assim como no Brasil, Uberlândia que é a segunda maior cidade do estado de Minas Gerais segue na mesma direção. Segundo o boletim de dados populacionais de 2019 do Centro de Estudos, Pesquisas e Projetos Econômico-Sociais (CEPES) da UFU, a taxa de crescimento anual da cidade no período de 2010 a 2018 foi de 1,55\%, bem superior aos demais municípios, que somados representaram um crescimento anual de $0,71 \%$ da população (BERTOLUCCI L, 2019). Tal crescimento pode relacionar-se, dentre outros fatores, à população estrangeira, já que Uberlândia ocupa a terceira posição em número de imigrantes no estado, como apontou o diagnóstico inédito sobre migração e refúgio em Minas Gerais (SOUZA DA, 2017). 
Dalmônica M (2018), relatou ao Jornal Diário de Uberlândia de 21/05/2018, que a Polícia Federal estimava à época, cerca de mil imigrantes residentes em Uberlândia, embora quatro mil já tivessem regularizado sua condição de estrangeiro. Silva VP (2017) apontou dificuldades em sua pesquisa quanto ao número real de imigrantes na mesma cidade, ao analisar os dados oficiais governamentais e não governamentais com foco no trabalho com imigrantes devido, principalmente, a rotatividade dessa população, o que torna difícil precisar esse quantitativo.

Importa saber que com o aumento da população imigrante no Brasil, faz-se necessário pensar como se dá o acesso a direitos humanos e constitucionais como: educação, saúde, alimentação, trabalho, moradia, lazer, segurança, previdência social, assistência dentre outros, estabelecidos pela Constituição Federal do Brasil (CF) de 1988, em seu Art. $6^{\circ}$, uma vez que em seu texto, no Art. 5ㅜ, a CF garante aos estrangeiros residentes no país, os mesmos direitos dos nacionais (BRASIL, 1988). Sobre a saúde, foco deste manuscrito, cumpre lembrar que um dos desdobramentos da CF/1988 foi a criação do Sistema Único de Saúde (SUS), por meio da Lei Orgânica da Saúde (LOS 8.080/90). Ministério da Saúde (1990) em cujo texto do Art. 2ํㅗ § 1ํㅡ, explicita o "acesso universal e igualitário às ações e aos serviços de saúde para a sua promoção, proteção e recuperação", sem qualquer distinção.

Nessa direção, a Lei de Migração oㅜ 13.445, vigente no país desde novembro de 2017, em substituição ao Estatuto do Estrangeiro, Lei no 6.815/1980, assegura ao migrante o "acesso a serviços públicos de saúde, (...) sem discriminação em razão da nacionalidade e da condição migratória” (Art. 3ำ - VIII). No mesmo ano de sua vigência, o Ministério da Saúde instituiu o Grupo de Trabalho sobre Saúde do Estrangeiro por meio da Portaria ํo 3.565 [20] de dezembro de 2017, com o intuito de assegurar e organizar as ações e serviços de saúde aos imigrantes, refugiados, residentes fronteiriços e visitantes no Brasil (BRASIL, 2017; BRASIL, 1980),

Apesar das legislações vigentes e da tentativa de organizar as ações voltadas a essa população, muitas são as barreiras para o acesso e atenção à saúde. Assim, o objetivo deste estudo foi objetivamos apresentar os entraves encontrados no atendimento à saúde de migrantes internacionais, em uma cidade de Minas Gerais.

\section{RELATO DE EXPERIÊNCIA}

As experiências aqui relatadas foram vivenciadas por estudantes de graduação, dos cursos de Medicina e Enfermagem, em um Hospital de Clínicas Universitário (HCU) e UBS do município. O maior número de migrantes atendidos era nacional do Haiti, do gênero feminino em idade ativa e, com maior demanda de atendimentos para ginecologia e obstetrícia. Chama a atenção que em sua maioria, as migrantes não trabalhavam, não tinham vínculo social e, apresentavam limitações na compreensão da língua portuguesa, o que dificultou sobremaneira a comunicação durante os atendimentos. Em alguns casos, as migrantes chegavam acompanhadas dos filhos em idade escolar, do parceiro, familiar ou amigo da mesma nacionalidade, para auxiliar na comunicação.

As experiências vivenciadas nos atendimentos permitiram identificar três pontos que merecem destaque, quais sejam: Falhas na comunicação como uma via de mão dupla; Perda de autonomia nos processos decisórios de saúde e, Inabilidade com competências culturais por parte dos profissionais da saúde, como segue.

\section{Falhas na comunicação como uma via de mão dupla}

A maior procura pelas UBS foi por razões de atendimento para pré-natal, porém, por terem dificuldade em se comunicar e entender as informações, as migrantes não comparecem a todas as consultas, não seguem as recomendações corretamente e, quando sentem algum sintoma específico, não conseguem explicar.

Uma das vivências emblemáticas foi a de uma haitiana que compareceu sozinha à unidade e apontava para a barriga sem falar nenhuma palavra em português. Nesse caso, o atendimento só foi possível porque após várias tentativas, outra haitiana percebeu a dificuldade de comunicação e se ofereceu para ajudar.

Também, um rapaz nacional do Haiti, sem falar português, necessitava realizar exames de sangue e, para se comunicar, utilizou como estratégia, um aplicativo de celular para tradução por voz, possibilitando a comunicação entre usuário-enfermeira. 
Observou-se também casos onde o atendimento não foi possível ou não respondeu bem às necessidades do usuário, como no atendimento de uma venezuelana recém-chegada ao Brasil, que compareceu à unidade para dar continuidade ao pré-natal iniciado em seu país. Apesar de portar o cartão de gestante do país de origem, optou-se por reiniciar o pré-natal com realização de todos os exames no Brasil, por incompreensão do idioma pela equipe de saúde e, da gestante, respectivamente.

Em outra situação, foi possível presenciar uma médica da unidade recusar atendimento a migrantes na ausência de um tradutor, o que não parece ser um caso isolado, uma vez que existiam queixas de alguns profissionais de saúde, por terem que prestar atendimento a pessoas sem conhecimento do seu idioma. Apesar de ser realidade a presença de vários migrantes internacionais na área de abrangência das UBS, percebeu-se que nem sempre os profissionais procuram desenvolver maneiras de acolher essa população.

Casos como os citados não são raros e, demonstram a dificuldade do profissional de saúde em atender o imigrante, assim como a dificuldade do imigrante em ser compreendido em suas necessidades de saúde, tornando o atendimento pouco ou nada resolutivo.

\section{Perda de autonomia nos processos decisórios de saúde}

A maioria das mulheres que chegam ao hospital é encaminhada de algum serviço de pré-natal para realizar o parto. Muitas delas chegam ao serviço de Ginecologia e Obstetrícia e não conseguem se comunicar em português, ficando a cargo dos maridos, que frequentemente já apresentam um domínio mediano da língua, realizar a tradução. Entretanto, ao contar com um tradutor que possui tal vínculo com o doente, torna-se limitada a atuação do paciente sobre suas próprias decisões.

Para exemplificar, citamos o caso de uma haitiana parturiente atendida no HCU acompanhada pelo marido que, por entender o português fazia todas as traduções. Quando a médica sugeriu um anticoncepcional para ser usado no puerpério, o marido não repassou a informação à esposa e respondeu prontamente, que a paciente não desejaria o anticoncepcional, não permitindo que a mulher soubesse e exercesse sua autonomia na tomada de decisão. Para além das questões de gênero e violência psíquica contra a mulher, esse caso ilustra o quão necessário se faz o entendimento do idioma do local de destino, para que a migrante seja protagonista de suas decisões nos processos de saúde.

\section{Inabilidade com competências culturais}

A dificuldade no relacionamento com o imigrante acentua as lacunas existentes na formação curricular dos profissionais de saúde, ao não incorporar as competências culturais, assim como na prática, não se faz presente tal temática nas capacitações e práticas profissionais, o que de certa forma, poderia reorientar a prática do cuidado. Essas fragilidades do conhecimento contribuem para gerar sentimentos de estranhamento perante a cultura do outro e dificuldade em entender seus hábitos.

Exemplificamos com o caso de uma imigrante, nacional de Bangladesh, em acompanhamento no ambulatório de pré-natal do HCU que apresentava constantemente picos de hipoglicemia, com risco ao bebê. A primeira dificuldade no contato surgiu na tentativa de explicar a necessidade de internação à família, pois, paciente e cônjuge não compreendiam bem o português. Ao ser internada, houve redução da hipoglicemia, porém, após a alta hospitalar e no seguimento ambulatorial, esse sinal voltou a aparecer. Era necessário entender, diante disso, sobre a alimentação doméstica, o que estava ligado não somente aos hábitos culturais, mas, também a condição de vida no Brasil. Porém, a equipe de saúde optou por não lidar com esse fato, por receio e descaso, além do desconhecimento da cultura do outro.

Outro caso vivenciado no HCU que demonstra como esse choque cultural pode causar discriminação, foi o de um rapaz de Moçambique, que procurou o HCU devido a um quadro gripal. Apesar de serem sintomas comuns, a conduta tomada pelo médico foi a de colocar o paciente em isolamento respiratório, cogitando que ele pudesse apresentar algum quadro respiratório grave, pelo fato de ser proveniente de um país africano.

Esses exemplos mostram o quanto é necessário avançar em capacitação e preparo dos profissionais da ponta, para que a legislação prescrita se cumpra na prática da garantia e acesso à saúde. Nenhuma condição idiomática e cultural, dentre outras, deve ser excludente do direito universal assegurado constitucionalmente. 


\section{DISCUSSÃO}

Apesar dos avanços inéditos desenhados na Lei da Migração brasileira, especialmente de acesso do imigrante aos serviços de saúde, percebe-se que na prática isso não aconteceu. Embora de caráter universal, no que tange aos imigrantes, o acesso à saúde nem sempre é possível, seja pela barreira do idioma de origem, seja pela questão cultural ou até mesmo pelo medo, em se tratando de imigrantes ilegais. Entretanto, nenhuma condição migratória pode ser impeditiva de acesso aos serviços de saúde (MARQUES MCC, et al., 2014).

A partir dos casos vivenciados na prática clínica, notou-se que o imigrante consegue acesso aos serviços de saúde, porém, questionamos a qualidade dos serviços prestados a essa população. Aguiar BS (2015) aponta que no estado de São Paulo a maioria dos imigrantes consegue ser atendida nas unidades de saúde e hospitais, entretanto, a barreira linguística e as diferenças culturais influenciam em falha de adesão ao tratamento na maioria dos casos. Além desses, outras dificuldades se fazem presentes como: discriminação e falta de informações sobre o SUS para os estrangeiros. Apesar das dificuldades, o Brasil possui algumas experiências exitosas quanto ao acesso em saúde aos imigrantes (CARNEIRO JÚNIOR N, et al., 2018).

São Paulo, que se destaca como a cidade que mais recebe fluxos migratórios no país criou em 2013 a Coordenação de Políticas para Migrantes, na Secretaria Municipal de Direitos Humanos e Cidadania. Em 2015, foi aprovado, pelo Conselho Municipal de Saúde, a Política Municipal de Saúde para Imigrantes e Refugiados, e, em 2016 foi aprovada a Lei no 16.478, que institui a Política Municipal para a População Migrante e, cria o Conselho Municipal de Imigrantes.

A partir da realização de oficinas, fóruns mensais e com a criação do Grupo de Trabalho de Saúde do Imigrante e Refugiado da Secretaria Municipal de Saúde de São Paulo, foram instituídas medidas para quebrar a barreira linguística, cultural e de discriminação no cuidado em saúde com o estrangeiro (CARNEIRO JÚNIOR N, et al., 2018; DOSP, 2016).

Além disso, foram contratados migrantes, à princípio bolivianos e, posteriormente de outras nacionalidades, como angolanos e haitianos para atuarem como agentes comunitários de saúde e agentes de prevenção de DST/AIDS, o que configura um grande avanço para a Estratégia Saúde da Família (ESF) (CARNEIRO JÚNIOR N, et al., 2011; SILVEIRA C, et al., 2014). Ganha relevo também a temática de atendimento ao imigrante implementada nos programas de Residência Médica e Multiprofissional em São Paulo com produção de folders confeccionados em sete idiomas sobre diversos problemas de saúde, entre eles dengue e tuberculose (CARNEIRO JÚNIOR N, et al., 2018).

Além de São Paulo, a Secretaria Municipal de Saúde do Rio de Janeiro, também vem tentando implementar políticas para a melhoria do acesso à saúde dos imigrantes (SMS/RJ, 2017). Por meio do livro Recomeços, são relatadas experiências implementadas para o atendimento aos refugiados. Em parceria com a Cáritas Arquidiocesana, organizou feiras de saúde com o objetivo de realizar ações de saúde sobre higiene bucal, doenças sexualmente transmissíveis e imunizações. Nessas feiras, era indicado aos imigrantes qual seria a sua unidade de saúde de referência e elaborada uma caderneta de saúde traduzida para o francês e o inglês a fim de facilitar o acesso em saúde.

Carneiro Junior N, et al. (2017), em seu estudo realizado em USB da cidade de São Paulo, mostrou que os profissionais da saúde utilizam de estratégias de desenho, mímicas e silabação de palavras com o intuito de reduzir as barreiras no atendimento aos imigrantes e gerar resolutividade no atendimento. Ou seja, a comunicação entre os profissionais que atuam na saúde e o usuário imigrante pode ter outras formas para além da fala do idioma, desde que haja interesse e busca recíprocos.

Assim, além do acesso, questionamos a qualidade dos serviços de saúde prestados aos migrantes internacionais, no tocante às demandas apresentadas e sua resolutividade. Ações como políticas públicas específicas para migrantes internacionais, contratação destes nos serviços de saúde, mudanças no processo formativo dos profissionais da saúde, para inserção de competências culturais e, capacitação profissional, nos parecem boas possibilidades como mecanismos de enfrentamento conjunto para garantir que os direitos humanos sejam cumpridos sem distinção de nacionalidade, em especial, o direito universal à saúde, foco desse manuscrito. 


\section{REFERÊNCIAS}

1. AGUIAR BS, et al. Alguns aspectos de saúde de imigrantes e refugiados recentes no município de São Paulo. São Paulo: Boletim CEinfo Análise, 2015; 52p.

2. BERTOLUCCI L. Dados populacionais: Análise Demográfica considerando as estimativas populacionais para 2018 dos municípios componentes da Mesorregião do Triângulo Mineiro e Alto Paranaíba -TMAP e das Regiões Geográficas Intermediárias de Uberlândia, Patos de Minas e Uberaba. Uberlândia: CEPES/IERIUFU, 2019; 40p.

3. BRASIL. Constituição Federal (1988). Constituição da República Federativa do Brasil. Brasília (DF):Senado Federal; 1988.

4. BRASIL. Lei n. 6.815, de 19 de agosto de 1980. Define a situação jurídica do estrangeiro no Brasil, cria o Conselho Nacional de Imigração. Disponível em: http://www.planalto.gov.br/ccivil_03/Leis/L6815.htm. Acesso em: 24 de maio de 2019.

5. BRASIL. Lei no 13.445, de 24 de maio de 2017. Institui a Lei de Migração. Diário Oficial da União. 2017. Disponível em: http://legis.senado.leg.br/legislacao/ListaTextoSigen.action?norma=17696552\&amp;id=17696557\&amp;idBinari o=17696561\&amp; mime=application/rtf\%20DOU\%2024\%20de\%20maio\%20de\%202017. Acesso em: 24 de maio de 2019.

6. CARNEIRO JUNIOR N, et al. Migração boliviana e doença de Chagas: limites na atuação do Sistema Único de Saúde brasileiro (SUS). Interface, 2017; 22(64): 87-96.

7. CARNEIRO JÚNIOR N, et al. Migração, exclusão social e serviços de saúde: o caso da população boliviana da cidade de São Paulo. BIS. Boletim do Instituto de Saúde (impresso), 2011; (13): 177-181.

8. CARNEIRO JÚNIOR N, et al. Políticas públicas nos contextos dos processos migratórios no Brasil. A experiência da construção da política municipal de saúde para imigrantes e refugiados na cidade de São Paulo (2015-2016). In: SILVEIRA C. GOLDBERG A. MARTIN D. (Org). Migração, Refúgio e Saúde. Santos: Ed. Leopoldianum, 2018; 320p.

9. COMITÉ NACIONAL PARA OS REFUGIADOS. Refúgio em Números. 4ํed. Brasília: Brasil, 2019; 46p.

10. ALMÔNICA M. Uberlândia é a 3a cidade com maior número de imigrantes em Minas. Jornal Diário de Uberlândia, 21/05/2018. Disponível em: https://diariodeuberlandia.com.br/noticia/16902/uberlandia-e-a-3--cidade-com-maiornumero-de-imigrantes-em-minas. Acesso em 5 de agosto de 2019.

11. DIÁRIO OFICIAL DA CIDADE DE SÃO PAULO. Lei no 16.478, de 8 de julho de 2016. Disponível em:http://diariooficial.imprensaoficial.com.br/nav_v4/index.asp?c=1\&gt. Acesso em: 5 de agosto de 2019.

12. DIÁRIO OFICIAL DA UNIÃO. Portaria ํㅡ 3.565, de 22 de dezembro DE 2017. Publicado em: 27/12/2017| Edição:247 | Seção: 1 | $\quad$ Página: 60 . Disponível em: http://www.in.gov.br/materia//asset_publisher/Kujrw0TZC2Mb/content/id/1392316/do1-2017-12-27-portaria-n-3-565-de-22-de-dezembro-de2017-1392312. Acesso em 5 de agosto de 2019.

13. FIGUEREDO LO, ZANELATTO JH. Legislação e políticas públicas voltadas à imigração no Brasil. Passagens Revista Internacional de História Política e Cultura Jurídica, 2016; 8(2): 252-274.

14. GOLDBERG A, et al. Por um campo específico de estudos sobre processos migratórios e de saúde na Saúde Coletiva. Interface; 2015; 19(53): 229-232.

15. INSTITUTO BRASILEIRO DE GEOGRAFIA E ESTATÍSTICA. Censo Demográfico 2010. Resultados gerais da amostra. $\quad$ Disponível http://www.ibge.gov.br/home/presidencia/noticias/imprensa/ppts/00000008473104122012315727483985.pdf. Acesso em: 5 de agosto de 2019.

16. INTERNATIONAL ORGANIZATION FOR MIGRATION. World Migration Report 2018. Geneva.

17. MARQUES MCC, et al. A atenção à saúde do imigrante no contexto histórico da saúde pública em São Paulo. In: MOTA A, MARINHO AGSMC, SILVEIRA C. Saúde e História de Migrantes e Imigrantes. Direitos, Instituições e Circularidades. São Paulo: Casa de Soluções e Editora, 2014; 232p.

18. BRASIL, MINISTÉRIO DA SAÚDE. Lei no 8.080, de 19 de setembro de 1990. Dispõe sobre as condições para a promoção, proteção e recuperação da saúde, a organização e o funcionamento dos serviços correspondentes e dá outras providências. Brasília, 1990.

19. OLIVEIRA AT. Um panorama da migração internacional a partir do Censo Demográfico de 2010. Rev. Interdiscip. Mobil. Hum, 2013; 21(40): 195-210.

20. PATARRA NL. Migrações internacionais de e para o Brasil contemporâneo: volumes, fluxos, significados e políticas. Perspec. [online]. 2005; 19(3): 23-33.

21. PATARRA NL. O Brasil: país de imigração? E-metrópolis. 2012; 9(3), 6-18.

22. RIBEIRO V. Coluna Direitos Humanos. Brasil já recebeu 1,1 milhão de imigrantes e 7 mil refugiados. Emissora Brasileira de Comunicação. Agência do Brasil Publicado em 19/01/2019. Disponível em: http://agenciabrasil.ebc.com.br/direitos-humanos/noticia/2019-01/brasil-ja-recebeu-11-milhao-de-imigrantes-e-7-milrefugiados Acesso em: 10 de março de 2019.

23. RIZEK CS, et al. Trabalho e imigração: uma comparação Brasil-Argentina. Lua Nova, 2010; 79:111-142.

24. SALADINI APS. Trabalho e imigração: os direitos sociais do trabalhador imigrante sob a perspectiva dos direitos fundamentais, Dissertação (Mestrado em Ciência Jurídica) -Centro de Ciências Sociais Aplicadas. Universidade Estadual do Norte do Paraná, Jacarezinho, 2011; 285 p.

25. SECRETARIA MUNICIPAL DE SAÚDE. Subsecretaria de Atenção Primária, Vigilância e Promoção da Saúde. Superintendência de Promoção da saúde. A experiência da Secretaria Municipal de Saúde do Rio de Janeiro no atendimento aos refugiados. 1 ed. Rio de Janeiro: SMS, 2017.

26. SILVA VP. Trabalhadores imigrantes na cidade de Uberlândia/MG: análise das políticas públicas brasileiras de trabalho e saúde no período de 2010 a 2016, Tese (Doutorado em Saúde Coletiva) - Departamento de Medicina Preventiva da Faculdade de Medicina. Universidade Federal de São Paulo, São Paulo, $2017 ; 128$ p.

27. SILVEIRA C, et al. Processos migratórios e saúde: uma breve discussão sobre abordagens teóricas nas análises em saúde dos imigrantes no espaço urbano. In: MOTA A, MARINHO AGSMC, SILVEIRA C. Saúde e História de Migrantes e Imigrantes. Direitos, Instituições e Circularidades. São Paulo, Casa de Soluções e Editora: 2014, 232p.

28. SOUZA DA. Diagnóstico sobre migração e refúgio em Minas Gerais. Belo Horizonte, UNILIVRECOOP: 2017, 467p. 